\title{
ON TOPOLOGICAL ENTROPY: WHEN POSITIVITY IMPLIES +INFINITY
}

\author{
SERGIĬ KOLYADA AND JULIA SEMIKINA
}

(Communicated by Yingfei Yi)

\begin{abstract}
In this paper we study the relations between the properties of the topological semigroup of all continuous selfmaps $S(X)$ on a compact metric space $X$ (the topological group $H(X)$ of all homeomorphisms on $X$ ) and possible values of the topological entropy of its elements. In particular, we prove that topological entropy of a functional envelope on the space of all continuous selfmaps on Peano continua or on compact metric spaces with continuum many connected components has only two possible values 0 and $+\infty$.
\end{abstract}

\section{INTRODUCTION AND MAIN RESULTS}

Let $X$ be a compact metrizable space and $S(X)$ be the set of all continuous maps from $X$ into $X$. Obviously, $S(X)$ is a topological semigroup under composition and the compact-open topology. Since $X$ is compact then, as is well known, the compact-open topology coincides with the topology of uniform convergence derived from a metric $\varrho$ compatible with the topology on $X$. Moreover, the latter topology is independent of the choice of the metric $\varrho$. Another topology on $S(X)$, equivalent to the two already mentioned, is the topology given by the Hausdorff metric. In fact, the maps in $S(X)$ have closed graphs and so we can consider a distance between elements of $S(X)$ defined as the Hausdorff distance (derived from, say, maximum metric in $X \times X)$ of their graphs. We will work with both of them in $S(X)$ : the uniform and the Hausdorff metrics.

By a topological dynamical system we mean a pair $(X, f)$, where $X$ is a metric (compact) space and $f: X \rightarrow X$ is a continuous map. The main topic of our research is to study the relations between the properties of the topological semigroup $S(X)$ (the topological group $H(X)$ of all homeomorphisms on $X$ ) and possible values of the topological entropy of its elements (continuous maps and homeomorphisms, respectively). More precisely, mostly we will consider the following two questions: 1) when does or does not a compact metric space admit a continuous map (homeomorphism) with positive topological entropy? 2) when does the existence of a positive-entropy continuous map on a compact metric space imply the existence of a $+\infty$-entropy continuous map?

If $A$ is a set in a metric space, by $B_{\varepsilon}[A]$ or $\bar{B}_{\varepsilon}[A]$ we will denote the union of all open or closed, respectively, balls of radius $\varepsilon>0$ whose centers run over the

Received by the editors March 9, 2013 and, in revised form, June 2, 2013.

2010 Mathematics Subject Classification. Primary 37B40; Secondary 54H20, 54H15.

The first author was supported by Max-Planck-Institut für Mathematik (Bonn); he acknowledges the hospitality of the Institute.

The second author was supported by Bonn International Graduate School in Mathematics. 
elements of $A$. If $A=\{a\}$, we write only $a$ instead of $\{a\}$, i.e., $B_{\varepsilon}(a)$ and $\bar{B}_{\varepsilon}(a)$. Let also $\partial B_{\varepsilon}(a):=\bar{B}_{\varepsilon}(a) \backslash B_{\varepsilon}(a)$.

Recall that the Hausdorff distance $d_{H}$ between two sets $A_{1}$ and $A_{2}$ in a metric space $X$ is given by

$$
d_{H}\left(A_{1}, A_{2}\right)=\inf \left\{\varepsilon>0: \bar{B}_{\varepsilon}\left[A_{1}\right] \supseteq A_{2} \text { and } \bar{B}_{\varepsilon}\left[A_{2}\right] \supseteq A_{1}\right\} .
$$

This is a metric on the family of all bounded, nonempty closed subsets of $X$. Note that if $X$ is compact we can apply this metric to (the graphs of) continuous maps from $X$ to $X$.

Given a compact metric space $(X, d)$, the set (group) $H(X)$ of all self-homeomorphisms of $X$, and the set (semigroup) $S(X)$ of all continuous maps from $X$ to $X$, we will consider the following metrics on these sets:

- The metric $d_{u}$ of uniform convergence (for $\left.H(X)\right): d_{u}(\varphi, \psi)=\sup _{x \in X}$ $\max \left\{d\left(\varphi^{-1}(x), \psi^{-1}(x)\right), d(\varphi(x), \psi(x))\right\}$. The corresponding space will be denoted by $H_{u}(X)$.

- The metric $d_{U}$ of uniform convergence: $d_{U}(\varphi, \psi)=\sup _{x \in X} d(\varphi(x), \psi(x))$. The corresponding spaces will be denoted by $H_{U}(X)$ and $S_{U}(X)$. Note that $d_{U}(\varphi, \psi)$ is well defined for any bounded selfmaps of $X$ (in fact, for any selfmaps of $X$, since $X$ is bounded).

- The Hausdorff metric $d_{H}$ (derived from the metric $d_{\max }\left(\left(x_{1}, y_{1}\right),\left(x_{2}, y_{2}\right)\right)=$ $\max \left\{d\left(x_{1}, x_{2}\right), d\left(y_{1}, y_{2}\right)\right\}$ in $\left.X \times X\right)$ applied to the graphs of maps (we identify a map and its graph, so we will write $\left.d_{H}(\varphi, \psi)\right)$. The corresponding spaces will be denoted by $H_{H}(X)$ and $S_{H}(X)$.

For any $\varphi, \psi \in H(X)$ we have $d_{H}(\varphi, \psi) \leq d_{U}(\varphi, \psi) \leq d_{u}(\varphi, \psi)$ and for any $\varphi, \psi \in S(X)$ we have $d_{H}(\varphi, \psi) \leq d_{U}(\varphi, \psi)$. If $X$ is a compact metric space, then the topologies given by the uniform metric and Hausdorff metric are equivalent in $H(X)$ and in $S(X)$.

In particular, a subset of $H(X)$ (of $S(X)$ ) is compact in $H_{u}(X)$ (in $S_{U}(X)$ ) if and only if it is compact in $H_{H}(X)$ (in $S_{H}(X)$ ). In general the two metrics are not uniformly equivalent (two maps, which are close to each other in the Hausdorff metric, may have a large distance in the uniform metric). Of course, on compact subsets of $H(X)$ (of $S(X)$ ) they are uniformly equivalent. Therefore $H_{u}(X)\left(S_{U}(X)\right.$ ) is compact if and only if $H_{H}(X)\left(S_{H}(X)\right.$, respectively) is compact. Finally, recall that the space $H_{u}(X)\left(S_{U}(X)\right)$ is complete, but in general not totally bounded and the space $H_{H}(X)\left(S_{H}(X)\right)$ is totally bounded but in general not complete and so in general none of them is compact.

Nevertheless, the compactness of the $S(X)$ and $H(X)$ is not a very strict condition and takes place quite often, because both of them may be very small. Recall that a topological space $X$ is said to be rigid, if the full topological homeomorphism group $H(X)$ is the identity. De Groot and Wille in [4] showed the existence of rigid plane locally connected one-dimensional continua (Peano curves). Later Cook in [3] constructed examples of (two) metric rigid continua such that: 1) the space of all continuous maps $S(X)$ (the full topological transformation semigroup) consists only of the constant maps and the identity; 2) the space of all homeomorphisms $H(X)$ (the full topological transformation group) is topologically equivalent to the Cantor set. But it is still an open problem. What can we say about the topological structure of the compact full topological homeomorphism group $H(X)$ and of the compact full topological homeomorphism group $S(X)$ (see the conjecture below). 
Recall that a topological group is called profinite group if it is Hausdorff, compact, and totally disconnected. Gartside and Glyn ([6]) have established that every metric profinite group is the full homeomorphism group of a continuum. Recently Hofmann and Morris in [8] proved that a compact full homeomorphism group of a Tychonoff space is a profinite topological group. But the following conjecture is still open (see [9] for details):

Conjecture 1. Let $G$ be a compact group. Then the following conditions are equivalent:

(1) There is a compact connected space $X$ such that $G \cong H(X)$.

(2) There is a compact space $X$ such that $G \cong H(X)$.

(3) $G$ is profinite.

If $(X, f)$ is a dynamical system given by a compact metric space $X$ and a continuous map $f: X \rightarrow X$, then the functional envelope of $(X, f)$ is the dynamical system $\left(S(X), F_{f}\right)$ whose phase space is the space of all continuous selfmaps of $X$ with the compact-open topology (considered as a metric space with the uniform metric or with the Hausdorff metric applied to the graphs of maps) and the map $F_{f}: S(X) \rightarrow S(X)$ is defined by $F_{f}(\varphi)=f \circ \varphi$ for any $\varphi \in S(X)$. This is one of the main notions in this paper and was first introduced in [1].

Note that the functional envelope of a system always contains an isometric copy of the original system - from this the name 'functional envelope' comes.

If $X$ is a compact metric space and $f: X \rightarrow X$ is continuous, then the map $F_{f}: \varphi \mapsto f \circ \varphi$ is uniformly continuous as a map $S_{U}(X) \rightarrow S_{U}(X)$, as well as a map $S_{H}(X) \rightarrow S_{H}(X)$ (see [1 for details). Hence the topological entropy (by the Bowen-Dinaburg definition; see Section 2) of $\left(S(X), F_{f}\right)$ is well defined for these metrics on $S(X)$. Since the functional envelope of a system always contains a copy of the original system, namely the constant mappings, we have $h\left(F_{f}\right) \geq h(f)$. The same inequality for the topological entropy of the functional envelope $\left(H(X), F_{f}\right)$ (i.e. the map $F_{f}(\varphi)=f \circ \varphi$ for a fixed $f \in H(X)$ and any $\varphi \in H(X)$ ) is not clear, and we do not know the answer in general.

Thus, if we denote the topological entropies of the systems $\left(S_{U}(X), F_{f}\right)$ and $\left(S_{H}(X), F_{f}\right)$ by $h_{U}\left(F_{f}\right)$ and $h_{H}\left(F_{f}\right)$, respectively, then $h_{U}\left(F_{f}\right) \geq h_{H}\left(F_{f}\right)$.

Theorem A. Let $X$ be a compact metric space. If $S(X)$ is compact, then for any $f \in S(X)$ topological entropy of $(X, f)$ and topological entropy the functional envelope $\left(S(X), F_{f}\right)$ is zero. If $H(X)$ is compact, then for any $f \in H(X)$ topological entropy of $(X, f)$ is also zero.

We will use here the term 'continuum' with two distinct meanings: the nondenumerable set of real numbers (set theory) and a nonempty, compact, metrizable, connected space (topology). A locally connected continuum is often called a Peano continuum.

It is well known that the topological entropy of a generic homeomorphism (continuous map) on a compact manifold with or without boundary (the dimension of which is greater than one) and the topological entropy of a generic continuous map on the interval is $+\infty$ (see 20] and 2] for details). In general we do not know when a compact metric space admits a continuous map (homeomorphism) with positive topological entropy and when this implies the existence of a continuous map (homeomorphism) with $+\infty$ topological entropy. Nevertheless we have the following. 
Theorem B. Let $X$ be a compact metric space with a nontrivial Peano subcontinuum or with continuum many connected components. Then $X$ admits a continuous map with infinite topological entropy, i.e., there exists a continuous map $f: X \rightarrow X$ with $h(f)=+\infty$.

Some examples of computing the topological entropy of the functional envelope $\left(S(X), F_{f}\right)$ can be found in [1] and in all of them the topological entropy takes one of the values $\{0,+\infty\}$. This observation gives rise to the following conjecture from [11.

Conjecture 2. Let $(X, f)$ be a topological dynamical system and $\left(S(X), F_{f}\right)$ its functional envelope. Then the only possible values of $h\left(F_{f}\right)$ are 0 and $+\infty$.

Progress on this conjecture was first done by Matviichuk in [13, who considered the case of the compact interval. He proved that zero topological entropy of the system $(I, f)$, where $f$ is a continuous selfmap of the interval $I$, implies zero topological entropy of its functional envelope $\left(S_{H}(I), F_{f}\right)$ (and it was known that positivity of topological entropy of $(I, f)$ implies infinite entropy of $\left(S_{H}(I), F_{f}\right)$ ). We describe a method of proving this conjecture for a certain class of spaces. In particular, it works in the case of an interval and the Cantor set.

Theorem C. Let $X$ be one of the following compact metric spaces: a Peano continuum or a space with continuum many connected components. Let $f$ be a continuous selfmap of $X$. Then the only possible values of $h\left(F_{f}\right)$ are 0 and $+\infty$.

\section{COMPACT HOMEOMORPHISM GROUPS AND CONTINUOUS SELFMAP SEMIGROUPS}

Recall the Bowen-Dinaburg definition of topological entropy (see [18]). Let $(Z, \varrho)$ be a metric space and $f: Z \rightarrow Z$ be uniformly continuous. For any integer $n \geq 1$ the function

$$
\varrho_{n}(x, y):=\max _{0 \leq j \leq n-1} \varrho\left(f^{j}(x), f^{j}(y)\right)
$$

defines a metric on $Z$ equivalent to $\varrho$.

Fix an integer $n \geq 0$ and $\varepsilon>0$ and let $K$ be a compact set in $Z$. A subset $E \subset K$ is called $(n, f, \varepsilon)$-separated, if for any two distinct points $x, y \in E, \varrho_{n}(x, y)>\varepsilon$. We say that a subset $F \subset Z(n, f, \varepsilon)$-spans the set $K$, if for every point $x \in K$ there exists a point $y \in F$ such that $\varrho_{n}(x, y) \leq \varepsilon$. Note that since $K$ is compact, $E$ is finite and $F$ may be infinite, but a finite subset that still spans $K$ exists.

Denote by $\operatorname{sep}(n, f, \varepsilon ; K)$ the maximal cardinality of an $(n, f, \varepsilon)$-separated set in $K$, and by $\operatorname{span}(n, f, \varepsilon ; K)$ the minimal cardinality of a set which $(n, f, \varepsilon)$-spans $K$.

For every $\varepsilon>0$ and $n \geq 0$ it holds

$$
\operatorname{span}(n, f, \varepsilon ; K) \leq \operatorname{sep}(n, f, \varepsilon ; K) \leq \operatorname{span}(n, f, \varepsilon / 2 ; K) .
$$

The topological entropy $h(f, K)$ on a compact set $K \subset Z$, is defined by

$$
h(f, K):=\lim _{\varepsilon \rightarrow 0} \limsup _{n \rightarrow \infty} \frac{1}{n} \log \operatorname{sep}(n, f, \varepsilon ; K)=\lim _{\varepsilon \rightarrow 0} \limsup _{n \rightarrow \infty} \frac{1}{n} \log \operatorname{span}(n, f, \varepsilon ; K) .
$$

Then the topological entropy $h(f)$ of a map $f: Z \rightarrow Z$ is defined by

$$
h_{\varrho}(f)=h(f):=\sup \{h(f, K): K \subset Z \text { and } K \text { is compact }\} .
$$

For uniformly equivalent metrics $\varrho_{1}$ and $\varrho_{2}$ we have $h_{\varrho_{1}}(X, f)=h_{\varrho_{2}}(X, f)$, and if $\varrho_{1}$ is stronger than $\varrho_{2}$, then $h_{\varrho_{1}}(X, f) \geq h_{\varrho_{2}}(X, f)$ (see [18]). 
We know that if $X$ is compact, then the map $F_{f}: \varphi \mapsto f \circ \varphi$ is uniformly continuous on both the spaces $S_{U}(X)$ and $S_{H}(X)$, and so we can study its topological entropy. Recall that $d_{U}\left(\varphi_{1}, \varphi_{2}\right) \geq d_{H}\left(\varphi_{1}, \varphi_{2}\right)$ for all $\varphi_{1}, \varphi_{2} \in S(X)$ and that these two metrics are equivalent on $S(X)$, hence uniformly equivalent on compact subsets of $S(X)$.

A system $(X, f)$ is called uniformly rigid if $f^{n_{i}}$ converges uniformly to $\mathrm{id}_{X}$ for some $n_{i} \rightarrow \infty$. The $\omega$-limit set of $x \in X$, denoted by $\omega_{f}(x)$, is the set of accumulation points of the forward orbit $\left\{f(x), f^{2}(x), \ldots, f^{n}(x), \ldots\right\}$. The identity map $X \rightarrow X$ will be denoted by $\operatorname{id}_{X}$.

Proof of Theorem A. Recall that $S_{U}(X)$ is compact if and only if $S_{H}(X)$ is compact. So, let us prove the theorem for $S(X)=S_{U}(X)$. Since space $S_{U}(X)$ is compact, by the Ascoli theorem (see [15, Chapter 7 in [10]) $S_{U}(X)$ is an equicontinuous family of maps on $X$. It means that for every $\varepsilon>0$ there is a $\delta>0$ such that for all $x, y \in X$ with $d(x, y)<\delta$ and all $\varphi \in S_{U}(X)$ we have $d(\varphi(x), \varphi(y))<\varepsilon$.

In particular, if $d_{U}\left(\varphi_{1}, \varphi_{2}\right)<\delta, \varphi_{1}, \varphi_{2} \in S_{U}(X)$, then for every $g \in S_{U}(X)$ it holds $d_{U}\left(g \circ \varphi_{1}, g \circ \varphi_{2}\right)<\varepsilon$. Hence the map $F_{f}$ is equicontinuous on the space $S_{U}(X)$ (i.e., the family $\left\{F_{f}, F_{f}^{2}, \ldots, F_{f}^{n}, \ldots\right\}$ is equicontinuous).

If a set is $\left(n, F_{f}, \varepsilon\right)$-separated, then it cannot contain points from $S_{U}(X)$ on a distance less than a corresponding $\delta$. Since $S_{U}(X)$ is compact, the cardinalities of such sets are bounded above by some constant depending only on $\delta$ (in fact depending on $\varepsilon)$ : $\operatorname{sep}\left(n, F_{f}, \varepsilon\right) \leq C(\varepsilon)$. It follows

$$
h\left(F_{f}\right)=\lim _{\varepsilon \rightarrow 0} \limsup _{n \rightarrow \infty} \frac{1}{n} \log \operatorname{sep}\left(n, F_{f}, \varepsilon\right) \leq \lim _{\varepsilon \rightarrow 0} \limsup _{n \rightarrow \infty} \frac{1}{n} \log C(\varepsilon)=0 .
$$

Therefore $0=h_{U}\left(F_{f}\right) \geq h_{H}\left(F_{f}\right) \geq h(f)$.

As we mentioned before, the case of a functional envelope $\left(H(X), F_{f}\right)$ if $f \in$ $H(X)$ is a bit more complicated. Formally there is no direct relation between topological entropies $h\left(F_{f}\right)$ and $h(f)$ in this case. If $H(X)$ is compact, then topological entropy of a functional envelope $\left(H(X), F_{f}\right)$ is zero (the proof is the same as for the case $\left.\left(S(X), F_{f}\right)\right)$.

Since $H(X)$ is compact, the $\omega$-limit set $\omega_{F_{f}}\left(\mathrm{id}_{X}\right)$ is nonempty. From Corollary 4.19 in 1 it follows that $(X, f)$ is uniformly rigid and therefore topological entropy $h(f)=0$.

\section{Theorem C For the interval And the CANTor SeT}

If $Z$ is a compact metric space and $T: Z \rightarrow Z$ is a continuous map, let $2^{Z}$ denote the space of closed subsets of $Z$ provided with the Hausdorff metric. It is well known that $2^{Z}$ is also a compact metric space, and there is a naturally induced action of $T$ on $2^{Z}$, defined by $T(A)=\{T(x) \mid x \in A\}$. Recall that a quasi-factor of the dynamical system $(Z, T)$ is a (closed) $T$-invariant subset of $2^{Z}$.

Let $(X, g)$ and $(Y, f)$ be dynamical systems, where $X$ and $Y$ are compact metric spaces, $g \in S(X), f \in S(Y)$. Consider the product system $(X \times Y, g \times f)$. Of course, the map $g \times f$ may be applied also to any subset of $X \times Y$, in particular, to graphs of continuous maps $X \rightarrow Y$. Denote by $\operatorname{gr}(\varphi)$ the graph of a map $\varphi \in C(X, Y)$, i.e., $\operatorname{gr}(\varphi):=\{(x, y) \in X \times Y: y=\varphi(x)\}$. The set $\operatorname{Gr}(X, Y):=\{\operatorname{gr}(\varphi): \varphi \in C(X, Y)\}$ of the graphs of all continuous maps from $X$ to $Y$ is a subset of $2^{X \times Y}$. When $g$ is a homeomorphism, one of the quasi-factors of the dynamical system $(X \times Y, g \times f)$ is the dynamical system $(\operatorname{Gr}(X, Y), g \times f)$. In particular, if $X=Y, g$ is the identity 


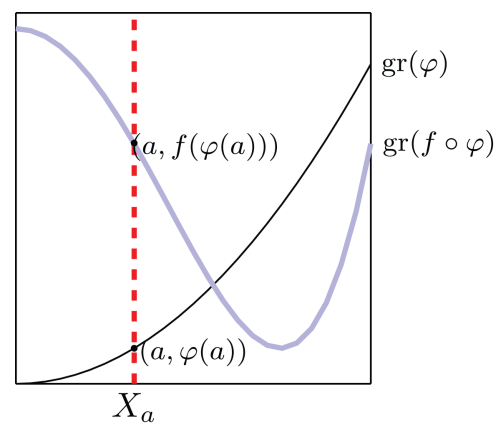

Figure 1. The dynamical system $\left(X^{2}, \mathrm{id}_{X} \times f\right)$ and a fiber $X_{a}:=$ $\left\{(x, y) \in X^{2}: x=a\right\}$. (Color available online.)

map on $X$ and we will make no distinction between maps and their graphs, then the system we get is the functional envelope $\left(S(X), F_{f}\right)$, where $F_{f}(\varphi)=f \circ \varphi$. So, formally the functional envelope $\left(S(X), F_{f}\right)$ is the same as the dynamical system $\left(\operatorname{Gr}\left(X^{2}\right), \mathrm{id}_{X} \times f\right)$, i.e., for any $\varphi \in S(X)$ the map $F_{f}$ maps each point $(x, y) \in$ $\operatorname{gr}(\varphi)$ into the point $(x, f(y)) \in \operatorname{gr}(f \circ \varphi)$ (in other words does not change the first coordinate; see Figure 1).

Let topological entropy of the map $F_{f}$ be equal to $\alpha$ and $0<\alpha<+\infty$. Then for any $\delta>0$ there is a compact subset $K \subset S(X)$ such that

$$
h\left(F_{f}, K\right):=\lim _{\varepsilon \rightarrow 0} \limsup _{n \rightarrow \infty} \frac{1}{n} \log \operatorname{sep}\left(n, F_{f}, \varepsilon ; K\right) \geq \alpha-\delta .
$$

We are going to show the existence of a precompact (with compact closure in the natural topology) subset $K_{K}$ of $S(X)$ with entropy at least twice larger than on $K$. We will do it for any compact subset of $S(X)$ and it does not depend on the space $S_{U}(X)$ or $S_{H}(X)$.

Recall that, since $X$ is a compact metric space, the uniform metric and Hausdorff metric are equivalent in $S(X)$ and uniformly equivalent on each compact subset of $S(X)$. Therefore a subset $K$ of $S(X)$ is compact in $S_{U}(X)$ if and only if it is compact in $S_{H}(X)$. It is also well known that a subset of $S(X)$, where $X$ is a compact metric space, has compact closure in a natural topology on $S(X)$ if and only if the subset is equicontinuous.

For much better understanding, we will first provide the proof of Theorem $\mathrm{C}$ for two cases: when $X$ is a compact interval or the Cantor set. Then we will use these important cases for the general one.

Theorem 3.1. Let $f$ be a continuous map on the interval I. Then the only possible values for the topological entropy $h\left(F_{f}\right)$ (it does not matter if $h_{U}\left(F_{f}\right)$ or $h_{H}\left(F_{f}\right)$ ) are 0 and $+\infty$.

Proof. Let $I:=[0,1]$ and divide this interval into three equal parts. In the first part we define a finite family of maps $T_{1}$ from $[0,1 / 3]$ to $I$ in the following way: $q \in T_{1}$ if $q(x)=\varphi(6 x), x \in[0,1 / 6]$ and $q(x)=\varphi(6(1 / 3-x)), x \in(1 / 6,1 / 3]$ for a $\varphi \in K$. In the third part we define a finite family of maps $T_{3}$ from $[2 / 3,1]$ to $I$ as follows: $g \in T_{3}$ if $g(x)=\psi(6(x-2 / 3)), x \in[2 / 3,5 / 6]$ and $g(x)=\psi(6(1-x)), x \in(5 / 6,1]$ for a $\psi \in K$. 

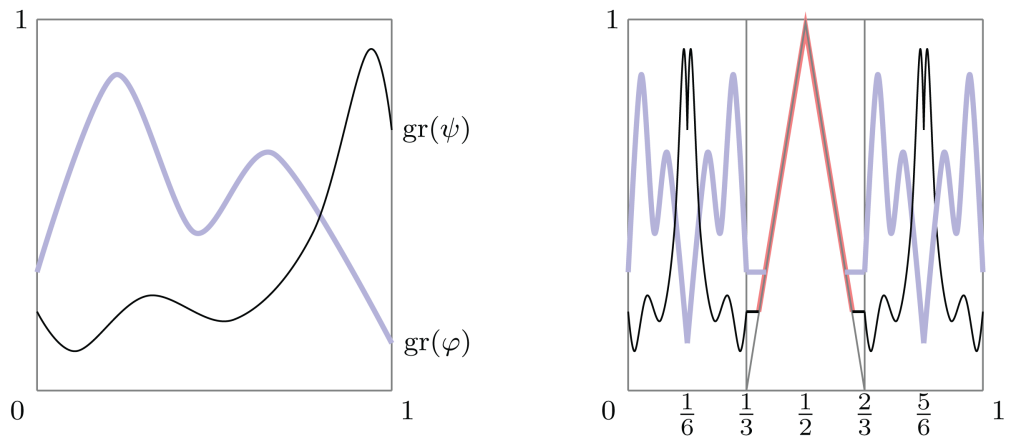

Figure 2. Graphs of 4 constructed maps from $K_{K}$. (Color available online.)

Now we "glue" each map from $T_{1}$ with any one from $T_{3}$ to get a continuous map on the whole interval $I$. We need just one additional assumption to all of these maps. They must be constants on small neighborhoods of points $1 / 3$ and $2 / 3$ inside of the interval $[1 / 3,2 / 3]$. For instance, it is shown in the picture (see Figure 2) by using a copy of the tent map $\tau(x)=6(x-1 / 3), x \in[1 / 3,1 / 2]$ and $\tau(x)=6(2 / 3-x), x \in(1 / 2,2 / 3]$.

So, we have defined the following sets of continuous maps $T_{1}:=\{g:[0,1 / 3] \rightarrow I$ : $g(x)=\varphi(6(x))$, if $x \in[0,1 / 6]$, and $g(x)=\varphi(6(1 / 3-x))$, if $x \in(1 / 6,1 / 3], \varphi \in K\}$ and $T_{3}:=\{q:[2 / 3,1] \rightarrow I: q(x)=\psi(6(x-2 / 3))$, if $x \in[2 / 3,5 / 6]$, and $q(x)=$ $\psi(6(1-x))$, if $x \in(5 / 6,1], \psi \in K\}$. Let also $\mathcal{A}:=\left\{(1 / 3, y) \in I^{2}: y=g(1 / 3), g \in\right.$ $\left.T_{1}\right\}$ and $\mathcal{B}:=\left\{(2 / 3, y) \in I^{2}: y=q(2 / 3), q \in T_{3}\right\}$. Consider the following family of tent maps:

$$
T_{\alpha, \beta}(x)= \begin{cases}\alpha, & x \in[1 / 3,1 / 3+\alpha / 6] ; \\ \tau(x), & x \in(1 / 3+\alpha / 6,2 / 3-\beta / 6] ; \\ \beta, & x \in(2 / 3-\beta / 6,2 / 3],\end{cases}
$$

where $0 \leq \alpha \leq 1$ and $0 \leq \beta \leq 1$. Define $T_{2}:=\left\{T_{\alpha, \beta}:(1 / 3, \alpha) \in \mathcal{A},(2 / 3, \beta) \in \mathcal{B}\right\}$. Put $K_{K}:=T_{1} \cup T_{2} \cup T_{3}$. Obviously $K_{K} \in S(I)$ and $T_{1}, T_{3}$ are compact by the definitions. Therefore $T_{1}$ and $T_{3}$ are also the equicontinuous sets. So, for the precompactness of $K_{K}$ the set $T_{2}$ must also be an equicontinuous set. But it is obviously true, because each map from $T_{2}$ is a continuous map which consists of "two constant maps" parts and "always the same tent map" part.

We are going to investigate both cases $S_{U}(I)$ and $S_{H}(I)$ for the $\left(n, F_{f}, \varepsilon ; K_{K}\right)_{-}^{-}$ separating properties.

- Case of $S_{U}(I)$. Fix a positive $\varepsilon$ and a positive integer $n$. Let $E=E(n, \varepsilon) \subset K$ be an $\left(n, F_{f}, \varepsilon ; K\right)$-separated subset with the cardinality $\operatorname{sep}(n, \varepsilon, K)$ (the maximal cardinality of the $\left(n, F_{f}, \varepsilon ; K\right)$-separated subsets of $\left.K\right)$. In the uniform metric two maps $\varphi$ and $\psi$ are $\left(n, F_{f}, \varepsilon\right)$-separated if there are an integer $0 \leq j \leq n-1$, and a pair of points $\left(x, f^{j}(\varphi(x))\right)$ and $\left(x, f^{j}(\psi(x))\right)$ (one point on each graph of the maps $f^{j} \circ \varphi$ and $f^{j} \circ \psi$, respectively) with the same first coordinate and sufficient large distance $(>\varepsilon)$ between the second coordinates.

For each map $g$ from $E$ there is a map $G \in K_{K}$, the graph of which has at least one compressed copy of $g$ (on the interval $[0,1 / 6]$ and/or $[2 / 3,5 / 6]$ ). Since 
the compression maps are $(x, y) \rightarrow(x / 6, y)$ and $(x, y) \rightarrow(x / 6+2 / 3, y)$ on these intervals, respectively, it does not change the second coordinates of graphs there. Therefore there exists a subset $E_{E} \subset K_{K}$ which is $\left(n, F_{f}, \varepsilon ; \overline{K_{K}}\right)$-separated and its cardinality is at least $\left(\operatorname{sep}\left(n, F_{f}, \varepsilon ; K\right)\right)^{2}$.

- Case of $S_{H}(I)$. Again fix a positive $\varepsilon$ and a positive integer $n$. Two maps $\varphi$ and $\psi$ are $\left(n, F_{f}, \varepsilon\right)$-separated in $S_{H}(I)$ iff there exists some $x \in[0,1]$ that the distance $d_{\text {max }}$ in $I \times I$ between the point $\left(x, f^{k} \circ \varphi(x)\right)$ on the corresponding graph and any point $\left(x^{\prime}, f^{k} \circ \psi\left(x^{\prime}\right)\right)$ on another graph is larger than $\varepsilon$. Hence, after the compression of these maps they will be $\left(n, F_{f}, \frac{\varepsilon}{6} ; \overline{K_{K}}\right)$-separated, because the distance between points may be less up to 6 times.

So, the sets $E_{E}$, which consist of at least $\left(\operatorname{sep}\left(n, F_{f}, \varepsilon ; K\right)\right)^{2}$ maps, are $\left(n, F_{f}, \varepsilon\right.$; $\left.\overline{K_{K}}\right)$-separated in $S_{U}(X)$ and $\left(n, F_{f}, \frac{\varepsilon}{6} ; \overline{K_{K}}\right)$-separated in $S_{H}(X)$, respectively. This means that entropy $h\left(F_{f}, \overline{K_{K}}\right)$ is at least twice larger than $h\left(F_{f}, K\right)$.

Hence, we can conclude that the only possible values for $h\left(F_{f}\right)$ are 0 and $+\infty$.

One can see that the proof for the case of the Cantor set is similar to the proof of Theorem 3.1, so we leave it to the reader.

\section{LoCALly CONNECTED CONTINUA}

Before we start to prove the main result of this section - Theorem $\mathrm{C}$, let us recall the following classical Hahn-Mazurkiewicz theorem: A nonempty Hausdorff topological space $X$ is a continuous image of the unit interval if and only if it is a compact, connected, locally connected metrizable space (i.e. Peano continuum).

Proof of Theorem C- the first part. Let $X$ be a locally connected metric continuum (with a metric $d$ ) and let $K$ be a compact subset of $S(X)$. Take a maximal cardinality $\left(n, F_{f}, \varepsilon ; K\right)$-separated set in $K$ and denote it by $E=E(n, \varepsilon)$. Now we are going to find a precompact set $K_{K} \subset S(X)$, which depends only on $K$ and has at least $\left(\operatorname{sep}\left(n, F_{f}, \varepsilon ; K\right)\right)^{2}$ of $\left(n, F_{f}, \varepsilon ; \overline{K_{K}}\right)$-separated maps for each positive integer $n$ and $\varepsilon>0$. We will do it similarly as in the proof of Theorem 3.1.

For a fixed point $x_{0} \in X$ let us define $r: X \rightarrow I$ by $r(x)=d\left(x_{0}, x\right)$. $\left(\max _{x \in X} d\left(x_{0}, x\right)\right)^{-1}$. Of course, the map $r$ is continuous and surjective. By the Hahn-Mazurkiewicz theorem there also exists a continuous surjective map $i: I \rightarrow X$.

Now, let $\varphi, \psi \in K$ and let $q(x)=\varphi(i(6 r(x)))$, if $r(x) \in[0,1 / 6]$ and $q(x)=$ $\varphi(i(6(1 / 3-r(x)))$, if $r(x) \in(1 / 6,1 / 3] ; g(x)=\psi(i(6(r(x)-2 / 3)))$, if $r(x) \in$ $[2 / 3,5 / 6]$ and $g(x)=\psi(i(6(1-r(x))))$, if $r(x) \in(5 / 6,1] ; \tau(x)=i(6 r(x)-2)$, if $r(x) \in[1 / 3,1 / 2]$ and $\tau(x)=i(4-6 r(x))$, if $r(x) \in(1 / 2,2 / 3]$.

For any pair $\varphi, \psi \in K$ we also define the following map:

$$
G_{\varphi, \psi}(x)= \begin{cases}q(x), & r(x) \in[0,1 / 3] ; \\ q\left(r^{-1}(1 / 3)\right), & r(x) \in(1 / 3, a] ; \\ \tau(x), & r(x) \in(a, 1 / 2] ; \\ \tau(x), & r(x) \in(1 / 2, b] ; \\ g\left(r^{-1}(2 / 3)\right), & r(x) \in(b, 2 / 3] ; \\ g(x), & r(x) \in(2 / 3,1],\end{cases}
$$




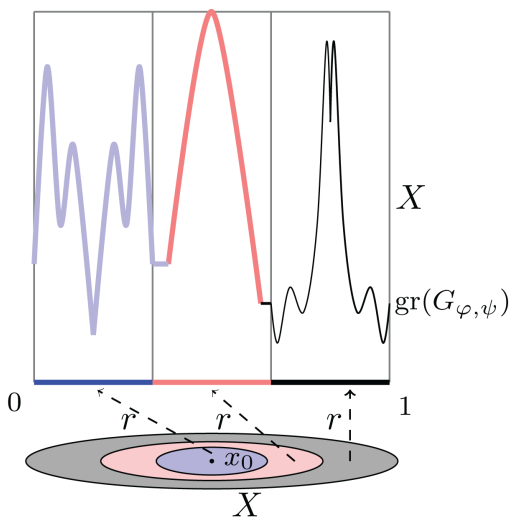

Figure 3 . (Color available online.)

where $a:=\min \{y \in[1 / 3,1 / 2]: i(6 y-2)=\varphi(i(0))\}$ and $b:=\min \{y \in[1 / 2,2 / 3]:$ $i(4-6 y)=\psi(i(0))\}$ (such values are nonempty, because $i$ maps $I$ onto $X$ ). Obviously, also $G_{\varphi, \psi}: X \rightarrow X$ is continuous (see Figure 3 ).

So, we can define $K_{K}$ as the following set $K_{K}:=\left\{G_{\varphi, \psi} \in S(X): \varphi \in K, \psi \in K\right\}$. It is easy to check that the set $K_{K}$ is equicontinuous and therefore precompact in $S(X)$.

Recall that the modulus of continuity of a continuous map $\Phi$ on the compact metric space $(X, d)$ is defined, with $\varepsilon>0$, as

$$
\omega(\varepsilon, \Phi):=\max _{x \in X} \max _{y \in B_{\varepsilon}(x)} d(\Phi(x), \Phi(y)) .
$$

Put $\Psi(x):=i(6(r(x)))$ for any $x \in X$. Since $\Psi \in S(X)$ is uniformly continuous on $X$, the modulus of continuity $\omega(\varepsilon, \Psi)$ tends to 0 when $\varepsilon \rightarrow 0$.

Let $E_{E}:=\left\{G_{\varphi, \psi} \in S(X): \varphi \in E, \psi \in E\right\}$. Obviously, the cardinality of $E_{E}$ is greater than or equal to $\left(\operatorname{sep}\left(n, F_{f}, \varepsilon ; K\right)\right)^{2}$. The set $E_{E} \subset K_{K}$ is $\left(n, F_{f}, \varepsilon ; \overline{K_{K}}\right)$ separated in $S_{U}(X)$ and $\left(n, F_{f}, \varepsilon_{\Psi} ; \overline{K_{K}}\right)$-separated in $S_{H}(X)$, where $\varepsilon_{\Psi}:=$ $\min \{\varepsilon ; \omega(\varepsilon, \Psi)\}$. This means that entropy $h\left(F_{f}, \overline{K_{K}}\right)$ is at least twice larger than $h\left(F_{f}, K\right)$. Therefore $h\left(F_{f}\right)$ is equal to $+\infty$ or 0 .

\section{Compact metric SPACES \\ WITH UNCOUNTABLY MANY CONNECTED COMPONENTS}

Before we start to prove the second part of Theorem $\mathrm{C}$ (for the case when $X$ is a compact metric space with continuum many connected components), let us provide all the tools we need.

Recall that a decomposition $D$ of a topological space $X$ is upper semi-continuous (u.s.c.) if for each element $E$ in $D$ and each open set $U$ containing $E$, there is an open set $V$ such that $E \subset V \subset U$ and $V$ is the union of members of $D$.

It is well known that every compact metric space is a continuous image of the Cantor set. We will also use the following inverse version of this fact:

Theorem 5.1. Every compact metric space $(X, d)$ with continuum many connected components can be continuously mapped onto the Cantor set. 
Proof. A decomposition of the space $X$ into connected components generates an equivalence relation on $X$. Let $Y:=X / \sim$ be the quotient space and $g: X \rightarrow Y$ be the quotient map. Given a compact metric space $X$ the decomposition of $X$ into components is upper semi-continuous and hence the quotient space $Y$ is a continuum (see Theorem 2.2 in [16]).

A locally compact Hausdorff space is zero-dimensional if and only if it is totally disconnected. $Y$ is a compact metric space, therefore it is also locally compact. As a consequence $Y$ is zero-dimensional. Since $Y$ is a separable metric space, all but countably many points of $Y$ are condensation points (i.e. each neighborhood of a condensation point contains uncountably many points) and the set of condensation points is perfect. Therefore the set of condensation points of $Y$ is homeomorphic to the Cantor set $C$. Sierpiński [17] showed that every nonempty closed subset of $C$ is a retract of $C$. This gives us the following well-known fact: every nonempty compact zero-dimensional metrizable space is homeomorphic to a retract of $C$. Since $Y$ is zero-dimensional, every nonempty closed subset of $Y$ is a retract of $Y$. Hence $C$ is a retract of $Y$.

Proof of Theorem C- the second part. The proof is almost the same as the proof of Theorem C - the first part, from Section 4. We just need to replace the map $i: I \rightarrow X$ by a continuous surjective map $i^{\prime}: C \rightarrow X$, where $C$ is the Cantor set, and the map $r: X \rightarrow I$ should be replaced by a continuous surjective map $r^{\prime}: X \rightarrow C$ (the existence of such a map follows from Theorem 5.1).

\section{Compact metric Spaces With $+\infty$-Entropy maps}

In this section we prove Theorem B. The proof will consist of two cases: 1) $X$ - compact metric space with a nontrivial Peano subcontinuum; 2) $X$ - compact metric space with continuum many connected components. In order to prove it, we need the following generalization of the commutativity of the entropy from [12.

Theorem 6.1. Let $X$ and $Y$ be compact topological spaces. For any continuous maps $f: X \rightarrow Y$ and $g: Y \rightarrow X$ the topological entropies $h(g \circ f, X)$ and $h(f \circ g, Y)$ are equal.

Proof. Since $f \circ(g \circ f)=(f \circ g) \circ f$, the map $f$ semiconjugates $g \circ f: X \rightarrow X$ with $f \circ g: f(X) \rightarrow f(X)$. The semiconjugacy $f: X \rightarrow f(X)$ is surjective and so $h(g \circ f) \geq h(f \circ g, f(X))$. Similarly, $h(f \circ g, f(X)) \geq h(g \circ f,(g \circ f)(X))$. Since $(g \circ f)(X) \subset X$ and $h(F, X)=h(F, F(X))$ for any continuous map $F: X \rightarrow X$, we get $h(g \circ f, X)=h(f \circ g, f(X))$.

Similarly one can show that $h(f \circ g, Y)=h(g \circ f, g(Y))$. So, we have $h(f \circ g, Y)=$ $h(g \circ f, g(Y)) \leq h(g \circ f, X)=h(f \circ g, f(X)) \leq h(f \circ g, Y)$. Hence $h(g \circ f, X)=$ $h(f \circ g, Y)$.

\section{Proof of Theorem B.}

Case 1. Recall that a space $Y$ is said to be arcwise connected provided that any two points of $Y$ can be joined by an arc in $Y$. It is known that every Peano continuum is arcwise connected (see Chapter VIII of [16]).

Let $P$ be a nontrivial Peano subcontinuum of the compact metric space $X=$ $(X, d)$. Take a point $x_{0} \in P$. Let us define the map $r: X \rightarrow I$ by $r(x)=$ $d\left(x_{0}, x\right) \cdot\left(\max _{x \in X} d\left(x_{0}, x\right)\right)^{-1}$, which is continuous and surjective. 


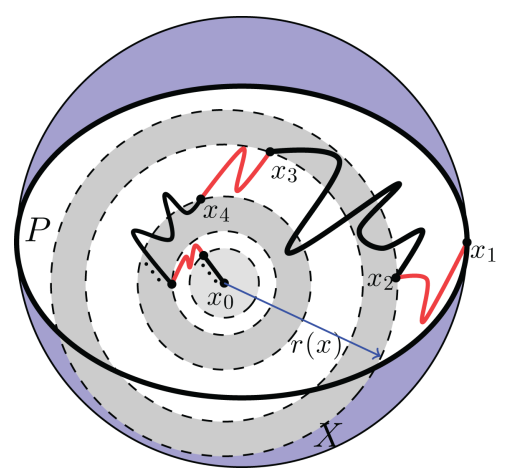

Figure 4. (Color available online.)

Now, take a point $x_{1} \in P$ and assume without loss of generality that $r\left(x_{1}\right)=1$. Since $P$ is arcwise connected there is an $\operatorname{arc} L \subset P$ which connects $x_{0}$ and $x_{1}$, i.e., a homeomorphism $\phi: I \rightarrow L$ such that $\phi(0)=x_{0}$ and $\phi(1)=x_{1}$.

Take a point $x_{2} \in L$ such that $x_{2}=\phi\left(\alpha_{2}\right)$, where $\alpha_{2}:=\max \{x \in$ $\left.\phi^{-1}\left(L \cap \partial B_{1 / 2}\left(x_{0}\right)\right)\right\}$. Let $L_{1}$ be the closed subarc of $L$ connecting points $x_{2}$ and $x_{1}$ and $J_{1}=\left[\alpha_{2}, \alpha_{1}\right]$, where $\alpha_{1}:=1$. Obviously, $\phi\left(J_{1}\right)=L_{1}$ and $r\left(L_{1}\right)=[1 / 2,1]$.

Let $x_{3} \in L$ be a point such that $x_{3}=\phi\left(\alpha_{3}\right)$, where $\alpha_{3}:=\min \{x \in$ $\left.\phi^{-1}\left(L \cap \partial B_{1 / 4}\left(x_{0}\right)\right)\right\}$. Take a point $x_{4} \in L$ such that $x_{4}=\phi\left(\alpha_{4}\right)$, where $\alpha_{4}:=$ $\max \left\{x \in \phi^{-1}\left(L \cap \partial B_{1 / 8}\left(x_{0}\right)\right)\right\}$. Let $L_{2}$ be the closed subarc of $L$ connecting points $x_{4}$ and $x_{3}$ and $J_{2}=\left[\alpha_{4}, \alpha_{3}\right]$. Obviously, $\phi\left(J_{2}\right)=L_{2}$ and $r\left(L_{2}\right)=[1 / 8,1 / 4]$.

Continuing in the same way we have the following sequence of points from $L$ : $x_{2 n-1} \in L$ is a point such that $x_{2 n-1}=\phi\left(\alpha_{2 n-1}\right)$, where $\alpha_{2 n-1}:=\min \{x \in$ $\left.\phi^{-1}\left(L \cap \partial B_{1 / 2^{2 n-2}}\left(x_{0}\right)\right)\right\} ; x_{2 n} \in L$ is a point $x_{2 n}=\phi\left(\alpha_{2 n}\right)$, where $\alpha_{2 n}:=\max \{x \in$ $\left.\phi^{-1}\left(L \cap \partial B_{1 / 2^{2 n-1}}\left(x_{0}\right)\right)\right\}$. Let $L_{n}$ be the closed subarc of $L$ connecting points $x_{2 n}$ and $x_{2 n-1}$ and $J_{n}=\left[\alpha_{2 n}, \alpha_{2 n-1}\right]$. Obviously, $\phi\left(J_{n}\right)=L_{n}$ and $r\left(L_{n}\right)=$ $\left[1 / 2^{2 n-1}, 1 / 2^{2 n-2}\right]$ (see also Figure 4 ).

Since $\phi: I \rightarrow L$ is a homeomorphism, the sequence $\alpha_{n}$ is converging to 0 when $n \rightarrow \infty$. Therefore we can define a homeomorphism $\varphi$ from $I$ onto $L$ such that $\varphi\left(1 / 2^{n-1}\right)=x_{n}$ for any $n=0,1,2, \ldots$. Let $I_{n}:=\left[1 / 2^{2 n-1}, 1 / 2^{2 n-2}\right]$. Then $\varphi\left(I_{n}\right)=L_{n}$ and as we already know $r\left(L_{n}\right)=I_{n}$.

Now we define a continuous map $g: I \rightarrow I$ with topological entropy $h(g)=+\infty$ in a standard way. Let $s \geq 2$. Recall that an $s$-horseshoe for a continuous map $f: I \rightarrow I$ is a closed interval $A \subset I$ and closed subintervals $A_{1}, \ldots, A_{s}$ of $A$ with pairwise disjoint interiors, such that $f\left(A_{j}\right)=A$ for $j=1, \ldots, s$. We shall denote this horseshoe by $\left(A ; A_{1}, \ldots, A_{s}\right)$. By the Misiurewicz theorem [14] the existence of an $s$-horseshoe for $f$ implies that topological entropy $h(f) \geq \log s$.

So, let $g$ be a continuous map which is piecewise monotone surjective (with $2 n+1$ pieces of monotonicity) on each closed interval $I_{n}$ and is the identity map on the interval between $I_{n+1}$ and $I_{n}, n \geq 1$. Since $g$ has a $2 n+1$-horseshoe for any $n \geq 1$, obviously we have that $h(g)=+\infty$ (see Figure 5 ).

So, we have defined the following continuous map $f:=\varphi \circ g \circ r$ from $X$ into itself, more precisely from $X$ onto $L$. We can rewrite this composition as the following 


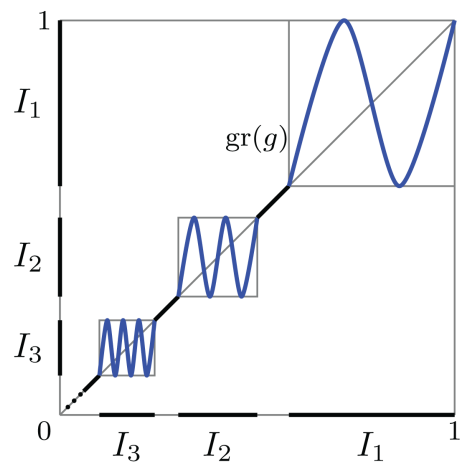

FiguRE 5 . (Color available online.)

(so-called) nonautonomous dynamical system

$$
X \stackrel{r}{\rightarrow} I \stackrel{g}{\rightarrow} I \stackrel{\varphi}{\rightarrow} L \stackrel{r}{\rightarrow} I \stackrel{g}{\rightarrow} I \stackrel{\varphi}{\rightarrow} L \stackrel{r}{\rightarrow} I \stackrel{g}{\rightarrow} \ldots \stackrel{\varphi}{\rightarrow} L \stackrel{r}{\rightarrow} I \stackrel{g}{\rightarrow} I \stackrel{\varphi}{\rightarrow} L \stackrel{r}{\rightarrow} \ldots
$$

Since we have the following inequality for topological entropy $h(f) \geq h\left(\left.f\right|_{L}, L\right)=$ $h(\varphi \circ g \circ r, L)$, instead we will consider the following periodic non-autonomous dynamical system:

$$
L \stackrel{r}{\rightarrow} I \stackrel{g}{\rightarrow} I \stackrel{\varphi}{\rightarrow} L \stackrel{r}{\rightarrow} I \stackrel{g}{\rightarrow} I \stackrel{\varphi}{\rightarrow} L \stackrel{r}{\rightarrow} I \stackrel{g}{\rightarrow} \ldots \stackrel{\varphi}{\rightarrow} L \stackrel{r}{\rightarrow} I \stackrel{g}{\rightarrow} I \stackrel{\varphi}{\rightarrow} L \stackrel{r}{\rightarrow} \ldots
$$

Using the commutativity of topological entropy (see Theorem 6.1), we can write this as $h\left(\left.f\right|_{L}, L\right)=h(\varphi \circ g \circ r, L)=h(r \circ \varphi \circ g, I)=h(g \circ r \circ \varphi, I)$. Since $\varphi\left(I_{n}\right)=L_{n}$ and $r\left(L_{n}\right)=I_{n}$ for any $n \geq 1$, the map $g \circ r \circ \varphi: I \stackrel{\text { onto }}{\rightarrow} I$ on the interval $I_{n}$ has an $s(n)$-horseshoe for any $n \geq 1$, where $s(n) \geq 2 n+1$. Therefore topological entropy $h(f) \geq h\left(\left.f\right|_{L}, L\right)=h(g \circ r \circ \varphi, I)=+\infty$.

Case 2. Although one can just repeat the proof of the previous case, we give here a somewhat different one. Let $i: X \rightarrow C$ be a continuous map from the space $X$ onto the Cantor set $C$. Such a map exists by Theorem 5.1. Take a map $g: C \rightarrow C$ with topological entropy $h(g)=+\infty$. Obviously such maps exist (see for instance [7]). As we have mentioned above, it is also well known that every compact metric space is a continuous image of the Cantor set. Let $\psi: C \stackrel{\text { onto }}{\rightarrow} X$ be such a continuous map. So, we have defined the following continuous map $f:=\psi \circ g \circ i$ from $X$ onto itself.

Using again the commutativity of topological entropy, we can write $h(f)=$ $h(f, X)=h(\psi \circ g \circ i, X)=h(i \circ \psi \circ g, C)=h(g \circ i \circ \varphi, C)$. Since $i \circ \varphi$ is a continuous (surjective) map from $C$ onto itself and $C$ is a compact metric space, it is easy to show that $h(g, C) \leq h(g \circ i \circ \varphi, C)$.

Really, let $(Y, T)$ be a dynamical system, given by a continuous map $T$ and a compact metric space $Y$, and let $S: Y \rightarrow Y$ be a continuous surjective map. Then for any $\varepsilon>0$, any positive integer $n$ and any (in particular, of maximal cardinality) $(n, T, \varepsilon)$-separated set $E$ in $Y$ there exists an $(n, T \circ S, \varepsilon)$-separated set $E_{S} \subset S^{-1}(E)$ in $Y$ with at least the same cardinality. So, obviously $h(T) \leq h(T \circ S)$. 
Therefore $h(f)=h(g)=+\infty$. As a concluding remark let us mention also that if in Case $1 X$ is a Peano continuum, then there also exists a continuous surjective map with $+\infty$ topological entropy.

\section{ACKNOWLEDGEMENTS}

The authors thank E. Bilokopytov, Y. Gutman, M. Matviichuk, D. Radchenko, L. Snoha and E. Tymchatyn for useful remarks and discussions.

\section{REFERENCES}

[1] Joseph Auslander, Sergii Kolyada, and Lubomír Snoha, Functional envelope of a dynamical system, Nonlinearity 20 (2007), no. 9, 2245-2269, DOI 10.1088/0951-7715/20/9/012. MR2351032(2008m:37012)

[2] L. S. Block and W. A. Coppel, Dynamics in one dimension, Lecture Notes in Mathematics, vol. 1513, Springer-Verlag, Berlin, 1992. MR1176513 (93g:58091)

[3] H. Cook, Continua which admit only the identity mapping onto non-degenerate subcontinua, Fund. Math. 60 (1967), 241-249. MR0220249 (36 \#3315)

[4] J. de Groot and R. J. Wille, Rigid continua and topological group-pictures, Arch. Math. 9 (1958), 441-446. MR0101514 (21 \#324)

[5] J. de Groot, Groups represented by homeomorphism groups, Math. Ann. 138 (1959), 80-102. MR0119193 (22 \#9959)

[6] Paul Gartside and Aneirin Glyn, Autohomeomorphism groups, Topology Appl. 129 (2003), no. 2, 103-110, DOI 10.1016/S0166-8641(02)00140-2. MR1961392 (2004g:54044)

[7] Christian Grillenberger, Constructions of strictly ergodic systems. I. Given entropy, Z. Wahrscheinlichkeitstheorie und Verw. Gebiete 25 (1972/73), 323-334. MR0340544 (49 \#5296)

[8] Karl H. Hofmann and Sidney A. Morris, Compact homeomorphism groups are profinite, Topology Appl. 159 (2012), no. 9, 2453-2462, DOI 10.1016/j.topol.2011.09.050. MR2921833

[9] K.H. Hofmann and S. A. Morris, Representing a Profinite Group as the Homeomorphism Group of a Continuum, arXiv:1108.3876v1 [math.GN], 2011.

[10] John L. Kelley, General topology, Springer-Verlag, New York, 1975. Reprint of the 1955 edition [Van Nostrand, Toronto, Ont.]; Graduate Texts in Mathematics, No. 27. MR0370454 (51 \#6681)

[11] Sergiı Kolyada, Topological dynamics: minimality, entropy and chaos (Ukrainian, with Ukrainian summary), Proceedings of Institute of Mathematics of NAS of Ukraine. Mathematics and its Applications, vol. 89, Institute of Mathematics of the NASU, Kiev, 2011. MR 3089342

[12] Sergiı̆ Kolyada and Ľubomír Snoha, Topological entropy of nonautonomous dynamical systems, Random Comput. Dynam. 4 (1996), no. 2-3, 205-233. MR1402417 (98f:58126)

[13] Mykola Matviichuk, Entropy of induced maps for one-dimensional dynamics, Iteration theory (ECIT '08), Grazer Math. Ber., vol. 354, Institut für Mathematik, Karl-Franzens-Universität Graz, Graz, 2009, pp. 180-185. MR2649016 (2011f:37028)

[14] Michał Misiurewicz, Horseshoes for mappings of the interval (English, with Russian summary), Bull. Acad. Polon. Sci. Sér. Sci. Math. 27 (1979), no. 2, 167-169. MR542778 (81b:58033)

[15] S. B. Myers, Equicontinuous sets of mappings, Ann. of Math. (2) 47 (1946), 496-502. $\operatorname{MR} 0017526(8,165 f)$

[16] Sam B. Nadler Jr., Continuum theory, Monographs and Textbooks in Pure and Applied Mathematics, vol. 158, Marcel Dekker Inc., New York, 1992. An introduction. MR 1192552 (93m:54002)

[17] W. Sierpiński, Sur les projections des ensembles complémentaires aux ensembles (A). Fund. Math., 11(1928), 117-122.

[18] Peter Walters, An introduction to ergodic theory, Graduate Texts in Mathematics, vol. 79, Springer-Verlag, New York, 1982. MR648108 (84e:28017)

[19] Gordon Thomas Whyburn, Analytic topology, American Mathematical Society Colloquium Publications, Vol. XXVIII, American Mathematical Society, Providence, R.I., 1963. MR0182943 (32 \#425) 
[20] Koichi Yano, A remark on the topological entropy of homeomorphisms, Invent. Math. 59 (1980), no. 3, 215-220, DOI 10.1007/BF01453235. MR.579700 (82b:58082)

Institute of Mathematics, NASU, Tereshchenkivs'ka 3, 01601 Kyiv, Ukraine

E-mail address: skolyada@imath.kiev.ua

Mathematical Institute of the University of Bonn, Endenicher Allee 60, 53115 Bonn, GERMANY

E-mail address: julia.semikina@gmail.com 